\title{
Japanese encephalitis virus: Uniqueness of immune response, vaccine development and future challenges
}

\author{
Milind M Gore, \\ National Institute of Virology, Gorakhpur Unit \\ BRD Medical College campus, Gorakhpur.
}

\begin{abstract}
Japanese encephalitis virus (JEV) is a major seasonal health problem in many rural areas in India and other parts of Asia. Transmission of virus is through mosquito vectors biting followed by peripheral multiplication site and exposed to host immune response before it succeeds in invasion of CNS. Thus protection from viral infection is a complex interplay of fight for superiority by virus and the host.
\end{abstract}

Cell mediated immune response using transferred to non-immune 14 day mice and lethally challenged to study the protection. Results indicated that dominant immune response of T helper (Th) 2 type. Th and neutralizing antibody inducing epitopes on JEV were identified by combination of immunological and Bioinformatics platforms. Chimeric peptides incorporating both Th and B cell epitopes could protect mice. These epitopes were further incorporated in polytope DNA construct with four chimeric peptides and induce protective immunity in mice. In addition, overcome the anergy development by traditional DNA vaccine plasmid than of CMV promoter using antigen specific cell promoter rather was also studied.

NIV carried out extensive studies on JE inactivated vaccines over the years. Studies were carried out mainly using CEC and Vero cells. Isolated of JEV from Kolar (821564) was extensively studied and thermostable mutant (821564-XY) was selected and characterized genetically as well as antigenically. A commercial successfully produced purified, inactivated vaccine JENVAC is licensed is being successfully. Future challenges in terms of single dose vaccine with long lasting immunity, pig immunization vaccine as well newer related flavivirus are also important.

Correspondence : Dr. Milind Madhukar Gore, National Institute of Virology, Gorakhpur Unit, BRD Medical College campus, Gorakhpur 273013 (UP).

DR. PRAN NATH CHHUTTANI ORATION delivered during NAMSCON 2015 at the All-India Institute of Medical Sciences, Patna. 


\section{INTRODUCTION}

An infection in animal has mediated trying to multiplication and growth and thus will have host- virus alternatives. A virus through entering mosquito to peripheral skin levels in Langerhans cells and peripheral antigen presenting cells (APCs) can understand large alternative approaches. Thus just inactivating cells, multiplication and cytokines and interferon levels to decrease, can increasing high levels of cells to pass through blood brain barrier junction or restriction and further in neuronal cells to damage further pathogenic even upto deceasing (1).

A Japanese encephalitis virus (JEV) is a vector-borne viral disease that occurs in South Asia, Southeast Asia, East Asia, and the Pacific. The disease can cause irreversible neurologic damage. The JE virus (JEV) is mainly transmitted by the mosquito Culex tritaeniorrhynchus, which prefers to breed in irrigated rice paddies. Wading ardeid water birds serve as virus reservoirs, but the virus regularly spills over into pigs, cattle and humans. Because infected pigs act as amplifying hosts, domestic pig rearing is an important risk factor in the transmission to humans. JE prevalence has been shown in these animals mainly by isolations and also by seroconversion. In India also, many studies have shown the dominance of Culex tritaeniorrhynchus as a major vector $(2,3)$.
Central nervous system (CNS) infections due to its preferred position and protection are rare, however, when any CNS abnormality happens it manifests as a very severe disease. In addition, residual damage due to loss of nervous tissue also leads to disabilities at various physical and mental levels. There are numerous medical conditions which may produce an encephalopathic illness; from traumatic to metabolic to immunopathological causes, which may mimic viral encephalitis. Encephalitis refers to an acute, usually diffuse, inflammatory process affecting the brain. While meningitis is primarily an infection of the meninges, a combined meningoencephalitis may also occur. An infection by a virus is the most common and important cause of encephalitis, although other organisms may sometimes cause an encephalitis. The time course of the viral encephalitis can be acute, subacute, or chronic. Pathologically there are encephalitis with direct viral entry into the CNS in which brain parenchyma exhibits neuronal damaging and viral antigens (4). The work of National Institute of Virology and our group in Immunology Division is being submitted as a comprehensive in the form of review. Brief in studies of basic peptide development, immunopathology studies, DNA vaccine have studied. Development of inactivated, tissue culture based, Indian based JEV vaccine in collaboration of NIV and private commercial agency has been launched since 2014 . 


\section{Immunopathological studies :}

\section{Innate immunity studies:}

During an infection, interplay between the host immune response and the virus like virulence factors, capacity to evade the host immune response, play an essential role in influencing the disease outcome. Innate immune response represents the first line of defense, and it is triggered at the first instance of pathogen exposure by peripheral antigen presenting cells (APCs) and innate lymphocytes $\mathrm{CD}^{+} 6^{+}$natural killer/natural killer $\mathrm{T}$ (NK/NKT) cells (5). On viral infection, these cells undergo maturation and activate a cascade of anti-viral immune responses as well as act as the scaffold for establishment of adaptive response. Macrophages and DCs are professional APCs, which on antigenic stimulation are functionally activated resulting in induction of surface co-stimulatory molecules, and cytokine and chemokine responses that are required for establishment of effective anti-viral response. Studies have also shown that viruses are capable of evading/ modulating the host immune response, depending on their virulence factors. Primary innate anti-viral response is established due to action of soluble mediators such as - type-I interferon (IFN), nitric-oxide (NO); and functional activation of APCs and $\mathrm{CD}^{+} 6^{+}(\mathrm{NK} / \mathrm{NKT})$ cells.

Two JEV strains JE057434 pathogenic, and SA14-14-2 were used in the study to understand the effect of viral virulence on the functional status of primary human monocytes derived macrophages (MDMs) and dendritic cells (MDDCs). Virus growth kinetic studies showed that MDMs support replication of both pathogenic and vaccine. Pathogenic $\mathrm{JEV}$ induced relatively lesser TNF $\alpha$ as compared to vaccine strain. The ability of JEV to induce type-I IFN from MDMs was dependent on virus dose and viral replication, rather than viral virulence. Transcript levels of OASs and RNaseL increased but remained identical between two strains at $18 \mathrm{hpi}$ and $24 \mathrm{hpi}$ in MDMs. However, Mx transcript increased in vaccine infected MDMs than pathogenic strain suggesting differential sensitivity towards Type-I IFN. Also, in order to determine whether pathogenic JEV infection mediates STAT1 degradation as compared to vaccine JEV, total STAT1 levels in JEV infected MDMs were determined at various time-points (i.e. 12, 24 and 48 hpi).

At lower IFN $\alpha$ concentration (10 $\mathrm{IU} / \mathrm{ml}$ ), IFN $\alpha$ pre-treatment resulted in complete abrogation of vaccine virus levels until later time points, however, in pathogenic JEV it was observed only for $24 \mathrm{hrs}$ which it recuperated subsequently. Based on these observations, it could be concluded that JEV strains are susceptible to anti-viral effects of IFNa, though the sensitivity varies. The vaccine strain is more sensitive to pre-established antiviral state than majority of the wild-type strains used in the study (6). 
Japanese encephalitis virus: Uniqueness of immune103

Interaction between DC and innate lymphocytes (i.e. CD56 ${ }^{+}$cells) represents a crucial event during anti-viral innate immune response. Apart from macrophages, DCs are known to support flavivirus replication. During viral infections, NK and NKT (CD56 ${ }^{+}$cells) are known to contribute to anti-viral response and aid in DC maturation. Presence of IL2-activated CD56 ${ }^{+}$cells enhanced imMDDCs maturation. Conversely, resting $\mathrm{CD}^{+} 6^{+}$cells were unable to modulate CD86 levels and most of the cytokine or chemokine tested, with an exception of IL6. Co-culturing of resting CD56 ${ }^{+}$cells led to an increased IL6 levels in uninfected and vaccine virus-infected imMDDCs cultures. It was also seen that IL2-activated $\mathrm{CD}^{+} 6^{+}$cells induced imMDDCs maturation partially through direct cell-to-cell contact and TNF $\alpha$, as separation of the cells by a permeable membrane or presence of neutralizing antibody against TNF $\alpha$ abrogated DC maturation. The IL2-activated $\mathrm{CD} 56^{+}$ cells showed higher degranulation (CD107a) capacity resulting in increased DC killing and greater ability to reduce JE viral load than resting $\mathrm{CD} 56^{+}$cells (7).

In conclusion, the data indicates that JEV interacts with MDMs/im-MDDCs at different levels and would depend on both viral and host factors. The viral virulence might be related with replication fitness and a decreased susceptibility to primary anti-viral response. Therefore, reduction in infectious virion production and increased sensitivity towards primary anti-viral response of vaccine JEV strain could facilitate efficient virus clearance and limiting viral spread into additional target tissues and thus aid in mounting up of beneficial immune response.

\section{Immunoprotective adaptive response:}

Once the virus is grown in cells and is destroyed from the cells adaptive immune response starts the mechanisms. Basic start rules are, APCs cells they took presenting killed or live virion on the MHC type I and II antigens. Correlation with MHCs with the presenting to peptide to CD 4 and CD8 cells work the lymphnode cells. Activated lymphocytes T cells and B cells multiply and destroyed various CTL cells, antibody productions in form of $\operatorname{IgM}$ and $\operatorname{IgG}$ antibodies and cleared out the infected virions. Neutralizing antibodies of specific types are generated in blood and brains and make the cells long term protection by viruses.

In order understanding in model mice virus and lymphocytes in details are studied. Adoptive transfer studies were carried out by generating JEV immune splenocytes in adult BALB/c mice and transferring these primed effector cells into naïve 14-day-old recipient animals for survival analysis following lethal JEV infection. The contribution of CD4+ or CD8+ T cell subsets to protection was also examined by either depleting or isolating these subsets from JEV immune splenocytes before adoptive transfer and determining the survival rate in recipient mice. In addition, the nature of the $\mathrm{T}$ helper response in adoptively transferred mice was studied by analyzing the cytokine profile and antibody subtypes 
produced in response to JEV infection. Differential gene expression profiling of brains from adoptively transferred mice was also performed in order to identify critical genes responsible for the modulation of infection at the target site (8).

The adoptive transfer of JEV immune splenocytes into naïve 14-dayold recipient mice resulted in the protection of $95 \%$ of the recipients from peripheral JEV challenge. The survival rate was reduced when transferred cells were depleted of the CD4+ T cell population $34.62 \%$ survival. Correspondingly, increased protection was observed when JEV primed CD4+ isolated $\mathrm{T}$ cells were transferred, as compared to $\mathrm{CD} 8+$ isolated $\mathrm{T}$ cells $53.85 \%$ and $28.57 \%$ survival respectively. Concurrent with results indicating a definite role for $\mathrm{CD} 4+\mathrm{T}$ cells in protection from JEV infection, it was seen that the repeated in vitro stimulation of JEV immune splenocytes with a peptide representing a $\mathrm{T}$ helper epitope from the prM region of JEV, followed by adoptive transfer of these cells into naïve mice was capable of conferring immunity in these animals from subsequent lethal JEV challenge. In addition, transfer of splenocytes from peptide immunized animals was also capable of protecting naïve recipient mice from infection with $\operatorname{JEV}(9)$.

Real time PCR analysis of virus titres in the organs of mice receiving JEV immune splenocytes revealed similar kinetics of virus replication at peripheral sites as in lethally infected mice. Virus titres in the brains were, however, much lower in mice that received JEV immune cell transfer. This suggested that mortality from JEV infection was in some part due to virus load in the CNS of infected mice and associated immunopathology. Mice receiving JEV immune splenocytes had considerably reduced levels of IFN- $\gamma$ and TNF- $\alpha$ in the sera. In contrast, sustained expression of the Th2 cytokines, IL-4 and IL-5, was observed at all-time points post infection. Differential gene expression studies from the brains of mice that were protected from JEV infection revealed a considerable increase in the expression of immunomodulatory cytokines like IL-4, IL-10 and TGF $\beta$.

In conclusion, protection from lethal JEV infection in naïve 14-day-old mice involved a CD4+ $\mathrm{T}$ cell mediated, Th2 immune response. In mice receiving JEV immune splenocytes, faster kinetics of Th2 antibody production resulted in higher levels of JEV specific antibody in the sera, which probably helped to reduce virus load in the CNS. Reduced levels of proinflammatory cytokines like IFN- $\gamma$ and TNF- $\alpha$ in the sera of protected mice, combined with an increase in Th2 cytokines like IL-4 and IL-5 probably achieved an immunomodulatory effect that resulted in the enhanced survival of these animals.

\section{Vero cell derived inactivated Indian JE vaccine :}

Vaccination is the single most important measure to control Japanese encephalitis. It is recommended that JE 
Japanese encephalitis virus: Uniqueness of immune105

immunization of children should be continued according to established schedules in regions where this vaccine has already been successfully introduced, preferably as part of the national immunization programs. NIV has carried out extensive studies on JE inactivated vaccines over the years. Studies were carried out mainly using CEC and Vero cells. The unpurified formalin inactivated vaccine has been studied. The blood sample from encephalitic patient (Lx-9 yr. female) was collected in 1981 (Nov-Dec) and was processed for isolation of etiological agent. Which was further confirmed as Japanese encephalitis virus using complement fixation test and neutralization test. This was named as 821564. From this parent strain of JEV (821564-XZ) thermostable mutant $(821564-X Y)$ was selected and characterized genetically as well as antigenically. This thermostable mutant was analyzed further for vaccine development. Antibody response against various strains in India and $\mathrm{HI}$ titres of mouse serum prepared against different JEV strains was studied. Shelf life of the vaccine using thermostable strain of $821564 \mathrm{XY}$ was found better than the parent JEV 821564 XZ strain.

Development of Thermostable Mutant of JEV-821564XY lyophilized M.Br.Susp was under MOU was transferred to Bharat Biotech International Ltd for Vero cell adaptation and vaccine development. The strain got readily adapted to Vero cell and BBIL then successfully produced inactivated vaccine. The antigenic as well as genomic differences between the two variants of 821564 were analyzed using different techniques. BBIL produced Vero cell inactivated JE vaccine. Purified and inactivated virus vaccine that has passed all the production quality testing would be used at desired concentrations. Suspension of vaccine preparation in aluminum hydroxide adjuvant would be carried out as per the standard procedures of manufacturing. The approved dose of $\mathrm{E}$ protein content in JENVAC is $5 \mu \mathrm{g} /$ dose. The vaccine subjected to various regulatory tests and trials. The vaccine JENVAC is about to be licensed for use in humans.This patent application also covers another aspect, at specified concentrations, are added to stabilize the purified live Japanese encephalitis virus bulk during inactivation process simultaneously (10).

\section{Integrated studies at NIV leading to vaccine development :}

Generation of mouse monoclonal antibodies (MAbs) were generated and carried for envelope proteins. These originated escape mutants classifications of JEV and flaviviruses were analyzed. IgM ELISA diagnosed was developed for JEV and DEN assays. These were supplied all over India in Govt. Health Systems since more than 7000 kits each year. There are MAbs derived JEV detection methods have also been worked.

The project was a continuation of collaborative efforts between Bioinformatics Centre, Department of Biotechnology Pune University and 
National Institute of Virology was studied as basic studies. While 3D envelope in silico and we could develop various peptides that could reacted with MAbs. This related B epitopes by synthesis peptides that were JEV neutralizing peptides.

As a part of Bioinformatics sciences of primary proteins again in silico in analysis $\mathrm{T}$ helper epitopes for Envelope and NS-1 and NS-3 of JEV was synthesized. Using immune splenocytes we could analysis good $\mathrm{T}$ helper peptides also. As both combining $\mathrm{T}$ helper and $\mathrm{B}$ cells peptides called - chimeric T helper B cells epitopes were synthesized, immunized in mice and could lethal challenge. Single chimeric peptide could protect mice protection also (11-13).

During these studies DNA vaccine was known to use for envelope etc. We also using combinations of four chimeric epitopes together in DNA plasmids. Therefore, it became imperative to design a construct containing multiple epitopes (polytope) as it would be a superior vaccine candidate for further enhancement of the immune response. Adjacent epitope units in the polytope construct were joined by highly flexible spacer sequences as linker, Polytope construct of JEV (P-JEV), was cloned inframe into pcDNA3.1/V5-His which is a transient expression vector(14). The protection observed following challenge of mice immunized by intramuscular route was $70 \%(15,16)$.

DNA vaccine offers an alternative strategy which can be further improved upon by incorporating various adjuvants. Successful immune response requires engagement of T cell receptor with MHCpeptide on professional APC as first signal. Simultaneously, second signal in the form of various co-stimulatory molecule engagements is necessary for sustained immune response. Failure to have this second signal may lead to reduced immune response or even anergy. Targeting DNA vaccine to APC has been studied. It can be argued that widespread expression of a gene through viral CMV promoter may lead to some adverse effect. Our attempt for immunomodulation was to target the antigen expression in professional APC by using the selective promoters. This could prevent anergy by expressing the antigen dominantly in professional APC. The constructs were further manipulated with the partial $\mathrm{C}$, prM and E (E) genes of JEV encoded by promoters of macrosialin (pMS-E), CMV (pCMV-E) and promoterless constructs (pNIX-E). Neutralizing antibody response to JEV showed that pCMV-E induced 1:450 titer while pMS-E induced 1:300, control plasmid and PBS did not show any detectable N'Ab titers. In summary, we have demonstrated herein the ex vivo expression of $\mathrm{E}$ protein directed by CMV and macrophage active promoter $(16,17)$.

\section{Future vaccine projects :}

Many newer developments using as basic studied already available can be used. In order to develop long term neutralized inactivated can be used. This means further using JENVAC inactivated 
Japanese encephalitis virus: Uniqueness of immune107

by adding $\mathrm{T}$ cells or chimeric peptides. Some basics are known results already present.

In order to develop DNA vaccines envelope along with chimeric epitopes in form of DNA vaccine for pigs can be used. These will be low cost pigs available. This will also means that before seasons we can immunized JEV infected and detecting amplified vaccine in field can also be worked. This even might decrease mosquito-JEV also.

During last few years both in Assam and Kerala human West Niles are being circulated in seasons. There is a need to be studied newer vaccines for humans at the earlier. As a part of inactivated WNV inactivated are needed based on Indian $\mathrm{WN}$ current viruses. In addition some studies showed that simultaneously CTL are also needed. It is possible that inactivated and CTL-T helper chimeric peptides together need to be incorporated.

NIV Scientifics along with Contributors and Collaborates over last about 60 years has some of the based inputs. I hope we start various things for our on Indian studies.

\section{REFERENCES :}

1. Myint KS, Kipar A, Jarman RG, et al. (2014). Neuropathogenesis of Japanese Encephalitis in a Primate Model. PLoS Negl Trop Dis 8: e2980. doi:10.1371
2. Gore MM (2014). Acute Encephalitis Syndrome in India: Complexity of the Problem. $J$ Commun Dis 46: 35-49.

3. C D C JEV fact sheet/http://www.cdc.gov/ncidod/dvbid/ jencephalitis/facts.htm.

4. King NJ, Getts RD, Getts TM, Rana S, Shrestha B, Kesson MA (2007). Immunopathology of flavivirus infections. Immunol Cell Biol 85: $33-42$.

5. Munz C, Steinman RM, Fujii S (2005). Dendritic cell maturation by innate lymphocytes: coordinated stimulation of innate and adaptive immunity. JExp Med 202: 203-207.

6. Sooryanarain H, Sapkal GN, Gore MM (2012). Pathogenic and vaccine strains of Japanese encephalitis virus elicit different levels of human macrophage effector functions. Arch Virol Arch 157:1905-1918.

7. Sooryanarain $\mathrm{H}$, Ayachit V, Gore $\mathrm{M}$ (2012). Activated CD56(+) lymphocytes $(\mathrm{NK}+\mathrm{NKT})$ mediate immunomodulatory and anti-viral effects during Japanese encephalitis virus infection of dendritic cells invitro. Virology 432 : 250-260.

8. Biswas SM, Kar S, Singh R, et al. (20 010$)$. Immunomodulatory cytokines determine the outcome of Japanese encephalitis virus infection in mice. $J$ Med Virol 82:304-310. 
9. Biswas SM, Ayachit VM, Sapkal GN, Mahamuni SA, Gore MM (2009). Japanese encephalitis virus produces a CD4+ Th2 response and associated immunoprotection in an adoptive-transfer murine model. $J$ Gen Virol 90 : 818-826.

10. Singh A, Mitra M, Sampath G, et al. (2015). A Japanese encephalitis vaccine from India induces durable and cross-protective immunity against temporally and spatially wide-ranging global field strains. $J$ Infect Dis 212 : 715-725.

11. Kutubuddin M, Kolaskar AS, Galande S, Gore MM, Ghosh SN, Banerjee K (1991). Recognition of helper $\mathrm{T}$ cell epitopes in envelope(E) glycoprotein of Japanese encephalitis, West Nile and Dengue viruses. Mol Immunol 28: 149-154.

12. Dewasthaly SS, Bhonde GS, Shankarraman V, Biswas SM, Ayachit VM, Gore MM (2007). Chimeric T helper- B cell peptides induce protective response against Japanese encephalitis virus in mice. Protein and Peptide Letters 14: 543-551.
13. Kutubuddin M, Gore MM, Banerjee K, Ghosh SN, Kolaskar AS (1993). Analysis of computer predicted antibody inducing epitope on Japanese encephalitis virus. Acta Virol 37: 417-428.

14. Kulkarni R, Sapkal G, Mahishi L, Shil P, Gore MM (2012). Design and characterization of polytope construct with multiple B and $\mathrm{T}(\mathrm{H})$ epitopes of Japanese encephalitis virus. Virus Res 166:77-86.

15. Kulkarni R, Sapkal G, Gore M (2012). Evaluation of Japanese encephalitis virus polytope DNA vaccine candidate in $\mathrm{BALB} / \mathrm{c}$ mice. Virus Research 170: 118-125.

16. Ahsan MF, Gore MM (2011). Comparative analysis of macrophage associated vectors for use in genetic vaccine. Virus Res 9:10-22.

17. Ahsan MF, Gore MM (2011). Comparison of immune response generated against Japanese encephalitis virus envelope protein expressed by DNA vaccines under macrophage associated versus ubiquitous expression promoters. Virol J 8:382-392. 